\title{
Serum D-Dimer Concentrations in Nephrotic Syndrome Track with Albuminuria, Not Estimated Glomerular Filtration Rate
}

\author{
D.J. Sexton ${ }^{\mathrm{a}}$ M.R. Clarkson ${ }^{\mathrm{a}}$ M.J. Mazur ${ }^{\mathrm{a}} \quad$ W.D. Plant ${ }^{\mathrm{a}} \quad$ J.A. Eustace ${ }^{\mathrm{a}, \mathrm{b}}$

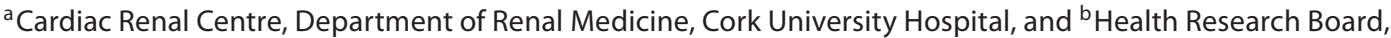 \\ Clinical Research Facility, University College Cork, Cork, Ireland
}

\section{Key Words}

Nephrotic syndrome $\cdot \mathrm{D}$-dimer $\cdot$ Thrombosis

\begin{abstract}
Background: The nephrotic syndrome is associated with an increased risk of venous and arterial thrombosis. There are little published data on the distribution, interpretation or determinants of serum D-dimer levels in patients with the nephrotic syndrome. We aimed to describe this relationship. Methods: This was a cross-sectional study of 100 patients with the nephrotic syndrome. Multivariate linear regression was used to evaluate for independent predictors of elevated D-dimer levels. Patients were observed for a period of 2 years after the baseline measurement of D-dimer level to assess for subsequent clinically evident thrombosis. Results: On univariate linear regression, D-dimer elevation was associated with age in years $\beta(95 \% \mathrm{Cl}) 0.02(0.016,0.03)$, log-transformed urinary protein:creatinine ratio in $\mathrm{g} / \mathrm{g} 0.439(0.32$, $0.558)$ and inversely with serum albumin in $\mathrm{g} / \mathrm{l}-0.05(-0.073$, -0.035 ) and estimated glomerular filtration rate (eGFR) in $\mathrm{ml} /$ $\mathrm{min} / 1.73 \mathrm{~m}^{2}-0.01(-0.016,-0.003)$. On multivariate linear regression, age in years $\beta(95 \% \mathrm{Cl}) 0.019(0.012,0.026)$, serum albumin in $\mathrm{g} / \mathrm{l}-0.023(-0.043,-0.003)$, and log-transformed urinary protein:creatinine ratio in $\mathrm{g} / \mathrm{g} 0.266(0.124,0.408)$
\end{abstract}

were independently associated with elevated D-dimer levels. Conclusion: D-dimer levels are commonly raised in the nephrotic syndrome in the absence of clinically evident thrombosis, and are independently associated with age, degree of proteinuria and serum albumin, but not with eGFR. Baseline levels of D-dimer did not predict subsequent episodes of clinically evident thrombosis after 2 years of follow-up.

Copyright $\odot 2012$ S. Karger AG, Base

\section{Introduction}

Nephrotic syndrome is characterised by heavy proteinuria and hypercholesterolaemia. It is associated with a significantly increased risk of venous thromboembolism, particularly deep vein and renal vein thrombosis [1-3]. Based on a retrospective cohort study of almost $300 \mathrm{pa}-$ tients with the nephrotic syndrome, the estimated annual incidence of venous thromboembolism is approximately $1.02 \%$ per year and $1.48 \%$ per year for arterial thrombosis $[4,5]$. Pulmonary embolism is estimated to be present in up to $32 \%$ of nephrotic patients presenting acutely with a serum albumin below $2 \mathrm{~g} / \mathrm{dl}$ [4]. Recently, a retrospective analysis of a large prospective cohort study described the prevalence of thromboembolism in patients with mem-

\section{KARGER}

Fax +4161306 1234

E-Mail karger@karger.ch

www.karger.com (c) 2012 S. Karger AG, Basel

0250-8095/12/0366-0554\$38.00/0

Accessible online at:

www.karger.com/ajn
Donal J. Sexton, MB, BCh, BAO, MRCPI, MD, BSc

Department of Renal Medicine, Cardiac Renal Centre

University College Hospital

Cork (Ireland)

E-Mail donalsexton0@ hotmail.com 
branous nephropathy [6]. Clinically apparent venous thromboembolic events occurred in 7\% of patients, and hypoalbuminaemia was the only independent predictor of thrombosis, with the risk of thrombosis increasing 2.13-fold for each gram reduction in serum albumin [6].

$\mathrm{D}$-dimer antigen is a fibrin degradation product released from cross-linked fibrin by the action of plasmin [7]. However, elevated levels of D-dimer do not always signify thrombosis, and many conditions can lead to initiation of intravascular fibrin formation without the development of overt thrombosis [7].

The clinical use of D-dimer measurement has been validated most extensively for the exclusion of venous thromboembolism [7]. In acute care settings, D-dimer has a high negative predictive value for excluding thrombosis; however, depending on the pre-test probability it may have a low positive predictive value [8]. In addition, the specificity of D-dimer for identifying thrombosis is reduced in the presence of comorbidities such as malignancy and infection [9].

There are sparse published data on the distribution and determinants of $\mathrm{D}$-dimer levels in patients with the nephrotic syndrome with which to inform nephrologists in attempting to interpret serum levels. One small study of 21 patients with the nephrotic syndrome without clinically evident thrombosis suggested increased activation of haemostasis in the nephrotic syndrome with increased levels of serum fibrinopeptide A, thrombin-antithrombin III complex and products of thrombin and prothrombin activation [10].

We aimed to define the distribution of $\mathrm{D}$-dimer levels and predictors of $\mathrm{D}$-dimer elevation in the nephrotic syndrome. Our hypothesis was that proteinuria might be associated with enhanced hepatic synthesis of fibrinogen in a similar manner to serum cholesterol in the nephrotic syndrome and hence $\mathrm{D}$-dimer elevation. Although previous studies have alluded to the relationship between reduced kidney function and D-dimer level [11], the relationship between proteinuria and D-dimer levels has not been thoroughly described. Secondly, we followed each patient in this cohort prospectively for 2 years to assess the incidence of thrombosis in those with and without elevated D-dimer levels at baseline.

\section{Methods}

We conducted a cross-sectional study on a convenience sample of 100 ambulatory non-hospitalised patients with the nephrotic syndrome attending the nephrology clinic after obtaining in- formed consent. Nephrotic syndrome was defined as proteinuria $\geq 3.5 \mathrm{~g}$ per $24 \mathrm{~h}$, oedema and hypoalbuminaemia ( $\leq 3 \mathrm{~g} / \mathrm{dl})$. All patients had been diagnosed with the nephrotic syndrome within 3 years of enrolment in this study. Patients in relapse, partial and complete remission and those who had never achieved a remission were included in our study, so that we could include a full range of degrees of proteinuria and severity of nephrotic syndrome. In order to comprehensively assess the association between $\mathrm{D}$-dimer levels and the explanatory variables, we needed to include patients with both active and inactive nephrotic syndrome.

All patients were assessed for symptoms suggestive of clinically evident deep venous thrombosis, pulmonary embolus, cerebral sinus thrombosis or renal vein thrombosis including: leg swelling, asymmetric leg swelling, calf discomfort, dyspnoea, chest pain, pleuritic chest pain, headache, blurred vision, or flank pain. Only patients without symptoms of thrombosis were included in the study. Patients were followed prospectively for a total period of 2 years after measurement of D-dimer levels. We measured serum $\mathrm{D}$-dimer levels on all prevalent $(\mathrm{n}=80)$ and incident $(\mathrm{n}=20)$ patients with the nephrotic syndrome. Laboratory data were collected cross-sectionally, and each patient was then followed prospectively for a minimum duration of 2 years after Ddimer measurement. All patients receiving anticoagulation $(\mathrm{n}=$ $10)$ and those with a clinically evident thrombosis $(n=1)$ at the time of enrolment were excluded from the study. Patients with a known diagnosis of malignancy or acute infection were also excluded from the study.

A clinically evident thrombosis was defined as any venous or arterial thrombosis that was discovered by the treating physicians as a result of direct investigation for thrombosis including pulmonary embolism, deep venous thrombosis, renal vein or cavernous sinus thrombosis, stroke or myocardial infarction.

Estimated glomerular filtration rate (eGFR) was calculated using the Modification of Diet in Renal Disease (MDRD 4 variable) equation and the Chronic Kidney Disease Epidemiology Collaboration (CKD-EPI) equations. The results of the analysis were not significantly different when either eGFR equation was used; therefore, data using the CKD EPI equation only are reported.

D-dimer assays used were the BIOPOOL MiniQuant ${ }^{\mathrm{TM}}$ and the Siemens INNOVANCE ${ }^{\mathrm{TM}}$ assay, which have been extensively validated for the diagnosis of venous thrombosis and pulmonary embolism [12-14]. Since both assays have different reference ranges, $\mathrm{D}$-dimer elevation was recorded by dividing the absolute D-dimer level by the upper limit of the reference range for each assay to generate fold elevations in reference range. These elevations were then $\log$ transformed. $73 \%$ of D-dimer levels were tested using the BIOPOOL assay, whereas $27 \%$ were analysed with the INNOVANCE assay.

Ethical approval was obtained from the medical research ethics committee at Cork University Hospital and University College Cork. Type one error was set at 5\%, which was two sided. SPSS version 20 was used to analyse the data. We conducted the regression analysis separately for both assays, and there was no significant difference in the results between assays.

We quantified the magnitude, significance and independence of the association of potential clinical predictors of serum D-dimer level including age, proteinuria and hypoalbuminaemia. We had an a priori hypothesis that a number of the explanatory variables would be associated with D-dimer levels. It is known that $\mathrm{D}$-dimer levels rise linearly with age, and it is also well established 
that GFR declines with age. Routine model diagnostics were undertaken.

Each explanatory variable, which was independently associated with elevated D-dimer level on univariate linear regression analysis, was included in the multivariate linear regression model $(\mathrm{p}<0.2)$. However, two of the explanatory variables, age and eGFR, were chosen a priori to be included in the multivariate model.

\section{Results}

We studied a total of 100 patients with the nephrotic syndrome. $61 \%$ were male. The mean (SD) age of our patients was 49.5 (19) years, eGFR (CKD-EPI) was 55.7 (28.1) $\mathrm{ml} / \mathrm{min} / 1.73 \mathrm{~m}^{2}$, serum albumin 3.3 (0.8) g/dl, median (25th-75th centile) protein:creatinine ratio was 2.3 (0.88-4.5) g/g. Median (25th-75th centile) C-reactive protein was $0.5(0-1)$. D-dimer levels were expressed as the D-dimer level divided by the upper limit of normal for the reference range of each assay. The median (25th75 th centile) $\mathrm{D}$-dimer elevation was $1(0.6,2.4) .17 \%$ had nephrotic syndrome due to diabetic nephropathy and $83 \%$ of patients had a primary glomerulonephritis. Of those with glomerulonephritis, $28 \%$ had primary focal segmental glomerulosclerosis, 19\% membranous nephropathy, $12 \%$ minimal change nephropathy, 9\% IgA nephropathy, $4 \%$ mesangiocapillary glomerulonephritis, $3 \%$ lupus nephritis; the remaining 14\% included various other forms of glomerulonephritis.

Overall, 53\% of patients in this cohort had an elevated D-dimer level. 36\% were in full remission with protein:creatinine ratio below $1 \mathrm{~g}$ per gram. In those with proteinuria above $1 \mathrm{~g} / 24 \mathrm{~h}$ at the time of measurement, $69 \%$ had an elevated D-dimer level. Median (25th-75th centile) D-dimer elevation tended to increase with decrease in eGFR, and in CKD stage I was $1(0.49,2.4)$, stage II $0.87(0.38,1.24)$, stage III $1.01(0.61,2.5)$, stage IV 1.81 $(0.65,4.6)$ and stage V $2.34(1.67,5.1)$.

Protein:creatinine ratio levels and D-dimer elevation were $\log$ transformed. When the data were divided into tertiles of serum albumin, there was a statistically significant difference in the mean log-transformed D-dimer elevation across tertiles of serum albumin (ANOVA $\mathrm{p}<$ 0.001), with D-dimer increasing as serum albumin decreases. When we convert log-transformed D-dimer levels back to fold elevation, we see the median (25th-75th centile) fold elevation was $0.82(0.38,1.24)$ in the highest tertile of serum albumin, $1(0.63,1.82)$ in the middle tertile and $2.28(1,3.6)$ in the lowest tertile of serum albumin.
Table 1. Univariate associations between D-dimer elevation and explanatory variables $(n=100)$

\begin{tabular}{lccr}
\hline & $\beta$ & $95 \% \mathrm{CI}$ & $\begin{array}{l}\mathrm{p} \\
\text { value }\end{array}$ \\
\hline Age & 0.024 & $0.016,0.03$ & $<0.001$ \\
Serum albumin & -0.05 & $-0.073,-0.035$ & $<0.001$ \\
eGFR & -0.01 & $-0.016,-0.003$ & 0.004 \\
Urinary protein:creatinine & & & \\
$\quad$ ratio & 0.439 & $0.32,0.558$ & $<0.001$ \\
Sex & -0.052 & $-0.435,0.331$ & 0.789 \\
\hline
\end{tabular}

Table 2. Multivariate linear regression model

\begin{tabular}{lrcc}
\hline $\begin{array}{l}\text { Model 1 } \\
(\mathrm{n}=100)\end{array}$ & $\beta$ & $95 \%$ CI & $\begin{array}{l}\mathrm{p} \\
\text { value }\end{array}$ \\
\hline Age & 0.018 & $0.01,0.25$ & $<0.001$ \\
Sex & -0.176 & $-0.449,0.097$ & 0.204 \\
$\begin{array}{l}\text { log-transformed urinary } \\
\quad \text { protein:creatinine ratio }\end{array}$ & 0.258 & $0.106,0.411$ & 0.001 \\
$\begin{array}{l}\text { Serum albumin } \\
\text { eGFR (CKD-EPI) }\end{array}$ & -0.025 & $-0.046,-0.004$ & 0.022 \\
& -0.003 & $-0.008,0.002$ & 0.28
\end{tabular}

$\mathrm{R}^{2}$ adjusted for model 1 is $51.1 \%$; for model 2 which excludes eGFR, the $\mathrm{R}^{2}$ adjusted is $51.0 \%$.

When we categorise the cohort into tertiles of urinary protein:creatinine ratio, we find a statistically significant difference in the mean log-transformed fold elevation in D-dimer (ANOVA $\mathrm{p}<0.001$ ). When we convert logtransformed D-dimer elevation back to fold elevation of D-dimer, we see median (25th-75th centiles) of this fold elevation was $0.49(0.38,1.0)$ in the lowest tertile, 1.06 $(0.82,2.4)$ in the second tertile and $2.34(1,4.3)$ in the highest tertile of urinary protein:creatinine ratio.

On univariate linear regression, serum albumin was inversely associated with urinary protein:creatinine ratio $(\mathrm{r}=-0.66, \mathrm{p}<0.001)$ but not with age or eGFR. Urinary protein:creatinine ratio was also associated with age $(\mathrm{r}=$ $0.229 \mathrm{p}=0.02)$ and inversely with eGFR $(\mathrm{r}=-0.244, \mathrm{p}=$ $0.01)$. Age was also associated inversely with eGFR ( $\mathrm{r}=$ $-0.36, \mathrm{p}<0.001)$. The univariate associations of $\mathrm{D}$-dimer level with explanatory variables are presented in table 1. Table 2 shows the multivariate model demonstrating the independent association of $\mathrm{D}$-dimer level with the explanatory variables, which adjusts for age, sex, log-trans- 
Table 3. Final multivariate linear regression model using backward stepwise variable selection method

\begin{tabular}{lrcr}
\hline Explanatory variables & $\beta$ & $95 \%$ CI & p value \\
\hline Age & 0.019 & $0.012,0.026$ & $<0.001$ \\
$\begin{array}{l}\text { Serum albumin } \\
\text { log-transformed urinary }\end{array}$ & -0.023 & $-0.043,-0.003$ & 0.026 \\
$\quad$ protein:creatinine ratio & 0.266 & $0.124,0.408$ & $<0.001$ \\
\hline
\end{tabular}

$\mathrm{R}^{2}$ adjusted is $51 \%$.

formed urinary protein:creatinine ratio, serum albumin and eGFR.

Using backward stepwise variable selection multivariate linear regression, the final model consisted of age, serum albumin and protein:creatinine ratio (table 3 ). The assumptions of univariate and multivariate linear regression were upheld. There no were significant interactions between the main effect explanatory variables. There was no evidence of multicolinearity between the explanatory variables. Due to our a priori hypothesis, we decided to include protein:creatinine ratio and serum albumin in the multivariate linear regression model. Since previous reports in CKD suggest that eGFR may be independently associated with $\mathrm{D}$-dimer elevation, it was decided to leave eGFR in the model despite the very modest increase in $\mathrm{R}^{2}$ adjusted.

Longitudinal data are available on 30 patients who had three measurements of serum $\mathrm{D}$-dimer levels taken on average 6 months apart while attending clinic over the course of the study. On each occasion, they also had their eGFR, serum albumin and urinary protein:creatinine ratio measured at approximately the same time during the day. The median (25th-75th centile) change in urinary protein:creatinine ratio was $0.81(-2.75,3.09) \mathrm{g} / \mathrm{g}$. This variation in each patient's degree of proteinuria over this period was due to achievement of a number of complete and partial remissions. Reductions in proteinuria were typically associated with reductions in serum $\mathrm{D}$-dimer and increases in serum albumin, while increases in proteinuria were associated with the opposite relationship. Median (25th-75th centile) change in serum albumin was $2.5(-5,2)$. The median $(25$ th-75th centile) change in fold elevation of $\mathrm{D}$-dimer level was $0.19(-0.02,0.41)$. Mean (SD) change in eGFR was $5(2) \mathrm{ml} / \mathrm{min} / 1.73 \mathrm{~m}^{2}$. There was a linear correlation between the serum albumin, urinary protein:creatinine ratio and serum $\mathrm{D}$-dimer level on each occasion. Pearson's correlation coefficient $(\mathrm{r})$ was $\geq 0.48$

Serum D-Dimer Levels in the Nephrotic Syndrome and $p<0.001$ between serum albumin, protein:creatinine ratio and serum $\mathrm{D}$-dimer on each occasion. When analysing the correlation between serum albumin and protein:creatinine ratio separately on each occasion, the correlation coefficient was $\geq 0.72$ and $p<0.001$ each time. The correlation between eGFR and serum D-dimer level varied with $\mathrm{r}=-0.14,-0.17$ and $-0.2, \mathrm{p}=0.04,0.04$ and 0.03 ; however, the correlations of eGFR with serum albumin and urinary protein:creatinine ratio were not statistically significant on each occasion (all p $>0.05$ ).

Over the entire follow-up period of 2 years, 2 patients developed a clinically evident thrombosis with renal vein thrombosis in one and pulmonary embolism in both. One patient had frequently relapsing steroid-responsive minimal-change nephropathy, and the other membranous nephropathy. Baseline D-dimer levels were normal in both patients at baseline and both were in full remission. However, later in the course of follow-up, both patients relapsed clinically, and shortly afterward presented to the emergency department. At the time of clinically evident thrombosis, $\mathrm{D}$-dimer levels were markedly elevated (i.e. $\geq 6$ times the upper limit of normal). Although we cannot suggest generalizable results based on 2 patients, it is possible that the change in $\mathrm{D}$-dimer level from baseline may be useful clinically to increase suspicion of thrombosis, particularly with dramatic elevations. None of those patients with an elevated D-dimer level at baseline went on to develop a clinically evident thrombosis over 2 years of follow-up.

\section{Discussion}

Plasma D-dimers are specific final cross-linked derivatives, which associate with fragment $\mathrm{E}$ in a complex, and which are generated when the endogenous fibrinolytic system degrades fibrin $[15,16]$. The molecular weight of $\mathrm{D}$-dimer is approximately $180 \mathrm{kDa}$ [17], and has a half-life of approximately $8 \mathrm{~h}$, with plasma clearance via renal excretion and the action of the reticuloendothelial system [18]. It has previously been reported that serum fibrinogen levels are raised in the nephrotic syndrome [2]; however, although one might expect that this is accompanied by an increased activity of the fibrinolytic system, in fact reports suggest that plasmin-induced fibrinolysis is actually reduced in the nephrotic syndrome [2]. One might speculate that despite reduced activity of plasmin-induced fibrinolysis, the degree of hyperfibrinogenaemia may be sufficient to result in increased fibrin degradation overall and hence increased D-dimer. 
There are few published data on the use of D-dimer measurements in the nephrotic syndrome; however, there are data on the association between D-dimer levels and reduced eGFR. A cross-sectional analysis of the Multi Ethnic Study of Atherosclerosis [11] found that D-dimer was moderately associated with eGFR using the CKD EPI equation, with levels increasing by $5 \%$ for every $10 \mathrm{ml} /$ $\mathrm{min} / 1.73 \mathrm{~m}^{2}$ reduction in eGFR [11]. However, this study did not address urinary protein excretion or serum albu$\min [11]$.

Possible mechanisms explaining the association of lower eGFR and higher levels of haemostatic factors such as $\mathrm{D}$-dimer have not been extensively characterised to date [11]. Increased levels of smaller-molecular-weight haemostatic compounds such as soluble tissue factor may be explained by the direct effect of reduced renal clearance [11]. This is unlikely to explain the reduced clearance of the higher-molecular-weight substances such as D-dimer and factor VIII [11]. It is plausible that elevations in these markers relate to processes initiated by smaller molecules or perhaps impaired haemostatic enzyme activity induced indirectly by kidney dysfunction [11].

We did not find an independent association between D-dimer levels and eGFR (CKD-EPI) after adjusting for degree of proteinuria and hypoalbuminaemia. The lack of independent association of D-dimer level with eGFR may relate to the relatively preserved eGFR in our cohort. Alternatively, the association between eGFR and D-dimer in other studies may have been confounded by proteinuria and hypoalbuminaemia.

Recently, limitations in the use of estimating equations for GFR based on serum creatinine in patients with the nephrotic syndrome have been uncovered. Hofstra et al. [19] studied the use of estimating equations based on serum creatinine, and found GFR may be systematically overestimated. It has been suggested that hypoalbuminaemia is associated with increased renal tubular creatinine secretion and therefore a reduced serum creatinine and spuriously increased eGFR [19]. This observation may explain why we did not find an independent association between serum D-dimer levels and eGFR after the adjustment for serum albumin and degree of proteinuria in our multivariate analysis. It is plausible that eGFR is in fact inaccurate in hypoalbuminaemic proteinuric patients, whereas other studies assessing the relationship between D-dimer and GFR did not include many patients with severe hypoalbuminaemia and/or heavy proteinuria [11].

Our a priori hypothesis in this study was that serum $\mathrm{D}$-dimer in the nephrotic syndrome might be related to urinary protein loss. In a manner similar to cholesterol production in the nephrotic syndrome, fibrinogen synthesis is increased [2]. Therefore, since D-dimer is the final degradation product of fibrinogen, it is conceivable that D-dimer is related to the severity of nephrotic syndrome as assessed by serum albumin and albuminuria.

In addition to the association with overt thrombosis, evidence is emerging that continuous low-grade fibrinolysis may be a marker of atherosclerosis and may be useful in the prediction of cardiovascular events [20]. Large observational studies in the elderly have demonstrated that elevated D-dimer levels are independently associated with future myocardial infarction [21] and cardiovascular death [22]. Whether nephrotic patients with an elevated D-dimer level are subject to increased risk of cardiovascular events compared to those with a normal D-dimer is unknown.

$\mathrm{D}$-dimer levels are known to increase linearly with age likely as a result of accrued co-morbid conditions and elevated plasma fibrinogen levels [9]; we also found this association in our cohort of patients with the nephrotic syndrome [9].

The interpretation of $\mathrm{D}$-dimer levels in the clinical management of patients with the nephrotic syndrome has the added difficulty of an elevated pre-test probability of thrombosis. Our data suggest that the D-dimer is raised in $70 \%$ of ambulatory non-hospitalised patients with a urine protein:creatinine ratio of $\geq 1$ g per gram. We suggest that in clinical practice when interpreting $\mathrm{D}$ dimer levels in the nephrotic syndrome, one should be aware of the degree of proteinuria.

The clinical use of D-dimer in the nephrotic syndrome may be challenging. The utility of $\mathrm{D}$-dimer in excluding thrombosis is heavily dependent on the pre-test probability [7], which may be moderate to high in the nephrotic syndrome due to the associated prevalence of thrombosis. From our data, the probability of a completely normal $\mathrm{D}$-dimer level even in the absence of a clinically evident thrombosis is low in those with significant proteinuria. We suggest that a much larger study is necessary to more accurately define the reference range of D-dimer level in the nephrotic syndrome; this study should also ideally assess for asymptomatic thrombosis. This may allow prediction estimates for $\mathrm{D}$-dimer based on the independent predictor variables.

Only the D-dimer levels recorded in clinic on routine follow-up were used in our analysis. However, 2 patients had repeat $\mathrm{D}$-dimer levels recorded at the time of acute presentation suggestive of thrombosis. These $\mathrm{D}$-dimer levels were dramatically elevated ( $\geq 6$ times the upper 
limit of the reference range), and when compared to levels recorded as baseline as part of our study, both patients had $\geq 3$-fold increase in D-dimer level. One patient had renal vein thrombosis, and both had pulmonary emboli. Both patients had normal D-dimer levels at baseline measurement and were in clinical remission with a normal protein:creatinine ratio at that stage, but were experiencing a relapse prior to the episode of thrombosis. It is impossible to glean any robust conclusions from this data based on 2 patients. However, perhaps D-dimer elevation is more useful clinically when it is elevated beyond that expected for the degree of proteinuria, age and hypoalbuminaemia.

Perhaps it may be useful to measure baseline levels of $\mathrm{D}$-dimer when the patient has no signs or symptoms of clinically evident thrombosis so that it might be repeated during acute presentations suggestive of thrombosis for comparison. In line with our findings, a recent prospective study of 100 patients with membranous nephropathy found that the positive predictive value of $\mathrm{D}$-dimer level for detecting venous thromboembolism was only $69.4 \%$; perhaps if this study had included multiple measurements, the change in $\mathrm{D}$-dimer level from baseline may have had a higher positive predictive value [23]. However, this is merely speculation since we cannot make any firm conclusions or recommendations based on 2 people in our sample.

Our study has a number of limitations. It may be difficult to suggest that the results of our study are generalizable to all patients with the nephrotic syndrome; however, the condition is rare, and it is difficult to assemble a much larger sample on which to systematically measure D-dimer levels.

The analysis is based on one measurement of serum $\mathrm{D}$-dimer. However, 30 patients had three measurements of serum D-dimer levels taken on average 6 months apart while attending clinic over the course of the study. Although there was obvious variation in each patient's degree of proteinuria over this period, these changes were mirrored by direct changes in serum D-dimer levels and inverse changes in serum albumin of similar magnitude. There was a linear correlation between the serum albumin, urinary protein:creatinine ratio and serum D-dimer level on each occasion (all $r \geq 0.48$ ).

We did not attempt to exclude asymptomatic thromboembolism in this sample, and it is plausible that a number of patients included could have had an undetected clinically silent thrombosis, which is a further limitation of our study. However, what we can say with confidence is that if undetected thrombosis was present, it did not

lead to an acute symptomatic presentation within 2 years of follow-up. Yet, a number of patients could have experienced a clinically silent thromboembolic event. We did not include a control group of patients with CKD who were matched for eGFR but without proteinuria. However, our sample included patients in partial and complete remission as well as those in relapse, and therefore included patients with and without significant proteinuria and with very similar eGFR.

D-dimer may also be elevated in malignancy, and a number of the glomerular diseases may represent a paraneoplastic manifestation; however, it is highly unlikely that a large number of the cohort had an occult malignancy since this is relatively rare, and patients were followed for a period of 2 years [24].

In conclusion, our study demonstrates that D-dimer levels are very commonly mildly raised in ambulatory non-hospitalised patients with the nephrotic syndrome without clinical evidence of thrombosis. D-dimer level at baseline is not associated with the development of clinically evident thrombosis within 2 years of follow-up. Ddimer elevation in the nephrotic syndrome is independently positively associated with age and spot urinary protein:creatinine ratio, and inversely with serum albumin.

\section{Disclosure Statement}

The authors declare that they have no conflict of interest.

References

Am J Nephrol 2012;36:554-560
1 Llach F: Hypercoagulability, renal vein thrombosis, and other thrombotic complications of nephrotic syndrome. Kidney Int 1985;28:429-439.

-2 Singhal R, Brimble KS: Thromboembolic complications in the nephrotic syndrome: pathophysiology and clinical management. Thromb Res 2006;118:397-407.

-3 Crew RJ, Radhakrishnan J, Appel G: Complications of the nephrotic syndrome and their treatment. Clin Nephrol 2004;62:245259.

-4 Radhakrishnan J: Venous thromboembolism and membranous nephropathy: so what's new? Clin J Am Soc Nephrol 2012;7: 3-4.

5 Mahmoodi BK, ten Kate MK, Waanders F, Veeger NJ, Brouwer JL, Vogt L, Navis G, van der Meer J: High absolute risks and predictors of venous and arterial thromboembolic events in patients with nephrotic syndrome: results from a large retrospective cohort study. Circulation 2008;117:224-230. 
-6 Lionaki S, Derebail VK, Hogan SL, Barbour $\mathrm{S}$, Lee T, Hladunewich $\mathrm{M}$, Greenwald $\mathrm{A}, \mathrm{Hu}$ Y, Jennette CE, Jennette JC, Falk RJ, Cattran DC, Nachman PH, Reich HN: Venous thromboembolism in patients with membranous nephropathy. Clin J Am Soc Nephrol 2012;7:43-51.

$\checkmark 7$ Adam SS, Key NS, Greenberg CS: D-dimer antigen: current concepts and future prospects. Blood 2009;113:2878-2887.

-8 Geersing GJ, Janssen KJ, Oudega R, Bax L, Hoes AW, Reitsma JB, Moons KG: Excluding venous thromboembolism using point of care $\mathrm{D}$-dimer tests in outpatients: a diagnostic meta-analysis. BMJ 2009;339:b2990.

9 Kelly J, Rudd A, Lewis RR, Hunt BJ: Plasma $\mathrm{D}$-dimers in the diagnosis of venous thromboembolism. Arch Intern Med 2002;162: 747-756.

10 Chen TY, Huang CC, Tsao CJ: Hemostatic molecular markers in nephrotic syndrome. Am J Hematol 1993;44:276-279.

11 Dubin R, Cushman M, Folsom AR, Fried LF, Palmas W, Peralta CA, Wassel C, Shlipak MG: Kidney function and multiple hemostatic markers: cross sectional associations in the multi-ethnic study of atherosclerosis. BMC Nephrol 2011;12:3.
12 Gardiner C, Pennaneac'h C, Walford C, Machin SJ, Mackie IJ: An evaluation of rapid $\mathrm{D}$-dimer assays for the exclusion of deep vein thrombosis. Br J Haematol 2005; 128:842848

13 de Moerloose P, Palareti G, Aguilar C, Legnani C, Reber G, Peetz D: A multicenter evaluation of a new quantitative highly sensitive $\mathrm{D}$-dimer assay for exclusion of venous thromboembolism. Thromb Haemost 2008; 100:505-512.

14 Coen Herak D, Milos M, Zadro R: Evaluation of the innovance D-DIMER analytical performance. Clin Chem Lab Med 2009;47: 945-951.

15 Kario K, Matsuo T, Kobayashi H: Which factors affect high D-dimer levels in the elderly? Thromb Res 1991;62:501-508.

-16 Crippa L, D’Angelo SV, Tomassini L, Rizzi B, D’Alessandro G, D'Angelo A: The utility and cost-effectiveness of D-dimer measurements in the diagnosis of deep vein thrombosis. Haematologica 1997;82:446-451.

17 Rowbotham BJ, Carroll P, Whitaker AN, Bunce IH, Cobcroft RG, Elms MJ, Masci PP, Bundesen PG, Rylatt DB, Webber AJ: Measurement of crosslinked fibrin derivatives use in the diagnosis of venous thrombosis. Thromb Haemost 1987;57:59-61.

18 Hager K, Platt D: Fibrin degeneration product concentrations (D-dimers) in the course of ageing. Gerontology 1995;41:159-165.
19 Hofstra JM, Willems JL, Wetzels JF: Estimated glomerular filtration rate in the nephrotic syndrome. Nephrol Dial Transplant 2011;26: 550-556.

20 Gorog DA: Prognostic value of plasma fibrinolysis activation markers in cardiovascular disease. J Am Coll Cardiol 2010;55:27012709.

21 Cushman M, Lemaitre RN, Kuller LH, Psaty BM, Macy EM, Sharrett AR, Tracy RP: Fibrinolytic activation markers predict myocardial infarction in the elderly. The cardiovascular health study. Arterioscler Thromb Vasc Biol 1999;19:493-498.

22 Alehagen U, Dahlstrom U, Lindahl TL: Elevated $\mathrm{D}$-dimer level is an independent risk factor for cardiovascular death in out-patients with symptoms compatible with heart failure. Thromb Haemost 2004;92:12501258.

23 Li SJ, Guo JZ, Zuo K, Zhang J, Wu Y, Zhou CS, Lu GM, Liu ZH: Thromboembolic complications in membranous nephropathy patients with nephrotic syndrome - a prospective study. Thromb Res 2012;130:501-505.

24 Birkeland SA, Storm HH: Glomerulonephritis and malignancy: a population-based analysis. Kidney Int 2003;63:716-721. 